\title{
Optimal control to reduce emissions in gasoline engines: an iterative learning control approach for ECU calibration maps improvement.*
}

\author{
Danilo Caporale ${ }^{1}$, Luca Deori, Roberto Mura, Alessandro Falsone, Riccardo Vignali, Luca Giulioni, Matteo Pirotta
}

\begin{abstract}
Control of emissions in gasoline engines has become more stringent in the last decades, especially in Europe. This poses new and important problems in the control of complex nonlinear systems. In this work a preliminary investigation is conducted on the idea of exploiting Iterative Learning Control to optimize calibration maps that are commonly used in Engine Control Units of gasoline engines. In this spirit, starting from existing maps, we show how to refine them using a gradient-descent iterative learning control algorithm, considering additional constraints in the optimization problem. The outcome of this procedure is a control signal which can be integrated in a modified map. The performance of the proposed technique is validated on the provided training signal and crossvalidated on different reference signals. Simulation results show the effectiveness of the approach.
\end{abstract}

\section{INTRODUCTION}

The need to reduce $\mathrm{CO}_{2}$ emissions in gasoline engines is a challenge for the control system designer, and innovative methods are required to drive the engines at their performance limits, while reducing emissions and avoiding uncontrolled phenomena such as knocking and misfiring that may arise in such conditions. It is required to take into account all these aspects in the controller design procedure, see [5].

A gasoline engine can be seen as a complex nonlinear system where many dynamics are involved: air and fuel intake and exhaust flows, mechanical elements and electrical components. In order to design a good control system, a model of the system to be controlled is needed, and the possible lack of accuracy in the model description has to be compensated by means of robust control techniques. In recent years Model Predictive Controllers for engines have been tested to explicitly handle contraints but they require an accurate model description, which is not always available, to compute an effective optimal control law, see [7], [8]. Dynamical models for gasoline engines are readily available, often in the form of Mean Value Models [5]. The approach proposed in this paper makes use of model free techniques to avoid the need of explicitly compute these models, in particular we focues on the so-called Iterative Learning Control method.

The main component of engine control systems are calibration maps, which are obtained by minimizing a certain

\footnotetext{
*This work has been submitted for the Student Competition

${ }^{1}$ Danilo Caporale and all the other authors are $\mathrm{PhD}$ students at the Department of Electronics, Informatics and Bioengineering at Politecnico di Milano danilo.caporale@polimi.it
}

functional over every operating point or operating region, see [5], [6] and references therein. In this work it is shown how to further improve such calibration maps in terms of better torque tracking performance, less fuel consumption and constraints satisfaction.

In particular an optimal control problem to refine an apriori given control law is formulated and solved. The main advantage is that it requires only the possibility to run multiple experiments on the system.

Iterative Learning Control (ILC) is generally used to improve tracking performance over repetitive tasks, taking into account desired control objectives, see [2], [3], [4]. In this way, the modelization and control design effort is moved to an automated algorithm for optimal tuning. This has been done in the industry for decades, see [6], but to the best of our knowledge an ILC approach has not been tested yet in the engine control framework, although it can be considered as a viable approach.

In the spirit of a preliminary study on the application of ILC to engine control design, and considering the nominal conditions where the engine is given to operate, our attention is posed on the open-loop behavior of the system, instead of using a dynamical feedback controller. The obtained improved maps define a control law capable of satisfying the prescribed performances and constraints. In view of this the effort for the designer is moved from the mathematical description of the system to the mathematical description of the problem and the implementation of its solution.

The paper is organized as follows: in section II the control problem is formulated based on [1]. In section III the proposed approach is presented. In section IV the results of the proposed approach are shown and cross-validated with different reference torques. In section $\mathrm{V}$ the conclusions are drawn for this work and further possible developments are shown.

\section{Problem Formulation}

Consider a nonlinear dynamical system $\mathcal{S}$ of the form:

$$
\mathcal{S}:\left\{\begin{aligned}
\frac{d x(\tau)}{d \tau} & =\mathbf{f}(x(\tau), u(\tau)) \\
y(\tau) & =\mathbf{g}(x(\tau), u(\tau))
\end{aligned}\right.
$$

where $x \in \mathbb{R}^{n}$ is the state vector, $u \in \mathbb{R}^{m}$ is the control vector and $y \in \mathbb{R}^{p}$ is the output vector, all continuous time functions.

The control variables for the problem are specified in [1] as the throttle valve position $x_{T H}[\%]$, the spark advance 
$\phi_{S A}[C A D]$, and the Exhaust Gas Recirculation (EGR) valve position $x_{E G R}[\%]$, collected in the vector:

$$
u:=\left[\begin{array}{lll}
x_{T H} & \phi_{S A} & x_{E G R}
\end{array}\right]^{\prime}
$$

while the chosen outputs are the minimum required:

$$
y:=\left[\begin{array}{lll}
T & h & q_{f}
\end{array}\right]^{\prime},
$$

respectively the measured torque in $[\mathrm{Nm}]$, the knocking and misfiring signaling function $h$ [1] and the instantaneous fuel consumption $q_{f}[\mathrm{~kg} / \mathrm{s}]$. The aim of the competition is to minimize the cost function

$$
\mathcal{J}(u)=\int_{0}^{t_{f}} c_{f} q_{f}^{2}(s)+c_{T}\left(T^{0}(s)-T(s)\right)^{2} d s,
$$

which takes into account the total fuel consumption and the torque tracking error for a specific reference torque, denoted by $T^{o}$. The constraints enforce the inputs to keep within given proper ranges, and require to avoid knocking and misfiring. The optimization problem results as in equation (5):

$$
\begin{array}{cl}
\min _{u} & J(u) \\
\text { s.t. } & h(x, u)<0 \\
& u \in U,
\end{array}
$$

where $U$ is a convex subset of $\mathbb{R}^{m}$.

The optimal control problem is cast in a discrete time form to take advantage of the ILC method. Consider a generic nonlinear discrete time dynamical system as in (6)

$$
\mathcal{S}_{d}:\left\{\begin{aligned}
x(t+1) & =f(x(t), u(t)) \\
y(t) & =g(x(t), u(t))
\end{aligned}\right.
$$

over a finite time interval of length $N$, $t \in[0,1,2, \ldots, N-1]$. Problem (5) has been recast as in (7),

$$
\begin{aligned}
J(u)=\sum_{\tau=0}^{t_{f}} & {\left[c_{f} q_{f}^{2}(\tau)+c_{T}\left(T^{0}(\tau)-T(\tau)\right)^{2}+\right.} \\
& \left.+M \sum_{i=1}^{n_{h}} \sigma\left(h_{i}(t)\right)\right], \\
& u \in U,
\end{aligned}
$$

where $M>0$ is a constant, $h(t) \in \mathbb{R}^{n_{h}}$ and

$$
\sigma(\eta)=\left\{\begin{array}{cc}
0, & \eta<0 \\
1, & \eta \geq 0
\end{array}\right.
$$

Note that in $h(\cdot)$ only the dependency on time is considered as of interest here. Also note that the saturation bounds on the control $u$ have been taken into account explicitly by adding saturations on the output of the each control variable. Three calibration maps were provided as a possible initial solution: such maps shall be indicated here with $\mathcal{M}_{1}$ for the one from $T^{o}$ to $x_{T H}, \mathcal{M}_{2}$ from $T^{o}$ to $\phi_{S A}, \mathcal{M}_{3}$ from $T^{o}$ to $x_{E G R}$.

We assume that it is possible to perform experiments on the system in steady state conditions, this can be done by simulation on a computer model or running tests on a prototype system in a laboratory. The variables related to each test are labeled by the letter $k$, so that $k \in\left[1,2, \ldots, N_{k}\right]$ can be addressed to as the iteration counter and $N_{k}$ is the number of tests, or iterations, one performs. Hence, each variable can be addressed to via an iteration index and a

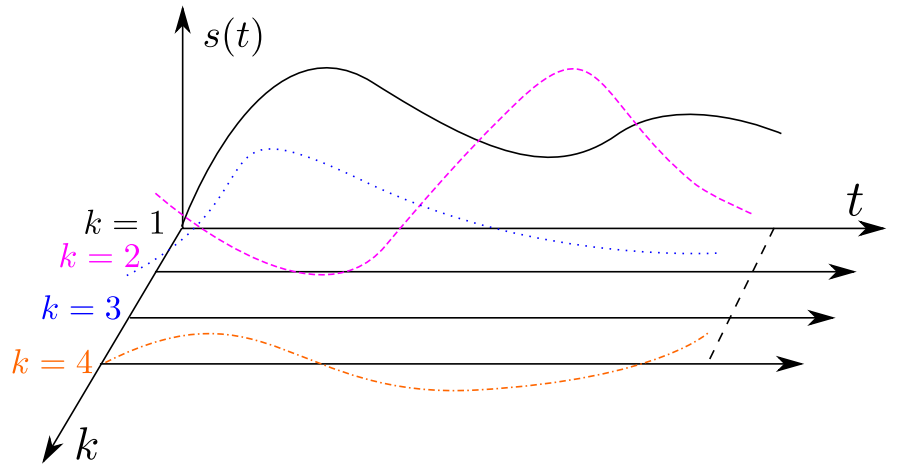

Fig. 1. Iterative Control Loop time axes: each signal $s_{k}(t)$ is defined over an iteration index $k$ and a time index $t$.

time index, see Figure 1. ILC is used to reduce the tracking error $e_{k}(t):=y_{k}^{o}(t)-y_{k}(t)$ between a reference signal and an output signal. For a SISO system, a common implemented law is the Arimoto's law [3]:

$$
u_{k}(t)=u_{k-1}(t)+\gamma e_{k}(t)
$$

The use of this proportional control law (which resembles an integral action between each iteration) has been proven to minimize the tracking error. A similar approach is used here where instead of the tracking error we aim at minimizing a figure of merit.

\section{Problem Solution Via Iterative LEARning CONTROL}

A common assumption for ILC algorithms is introduced.

Assumption 1: The process under control, when fed with the output of the preliminary calibration maps $\mathcal{M}_{i}, i=1 . .3$, is globally asymptotically stable.

The gradient-descent method has been chosen to minimize the figure of merit, hence an estimation of the gradient of $J(u)$ with respect to the control variables $u$ is needed. Let $u_{k}$ be the control signal given at a certain iteration $k$. To estimate the gradient one can perturb this signal on the next iteration as in:

$$
u_{k, j}(t)=u_{k}(t)+\delta u_{k, j}(t)
$$

where $j=1,2,3$ denotes which input we are perturbing and $\delta u_{j}(t)>0, \forall t \in[0,1,2, \ldots, N-1]$ is a perturbation of the control signal's $j-t h$ component. Then one can let $\nabla_{u} J_{k}\left(u_{k}\right)$ be the gradient of the function $J$ with respect to $u$ as in:

$$
\nabla_{u} J_{k}\left(u_{k}\right):=\left[\begin{array}{lll}
\frac{J_{k, 1}-J_{k}}{\delta u_{k, 1}} & \frac{J_{k, 2}-J_{k}}{\delta u_{k, 2}} & \frac{J_{k, 3}-J_{k}}{\delta u_{k, 3}}
\end{array}\right]
$$

Once the gradient has been estimated one can perform a gradient descent optimum search as shown in [2], which is done by the following control law:

$$
u_{k+1}(t)=u_{k}(t)-\alpha_{k} \nabla_{u} J_{k}\left(u_{k}\right)
$$

where $\alpha_{k}>0$ is the variable step length which takes into account the gradient normalization and the step adaptation. 
For the sake of clarity, we show here the algorithm used to find the optimal control maps:

Require: For $k=1$ run simulations with maps $M_{i}$.

for $k=2$ to $N_{k}$ do

Compute the gradient estimation as in (11).

for $j=1$ to $m$ do

Apply the control $u_{k, j}(t)=u_{k}(t)+\delta u_{k, j}(t)$

end for

Apply the control (12).

$J_{k} \leftarrow$ (7).

while $J_{k}>J_{k-1}$ do

$\alpha \leftarrow \alpha / 2$.

Apply the control (12).

$J_{k} \leftarrow$ (7).

end while

\section{end for}

In [2], the convergence of the solution to the optimum is shown, under suitable assumptions on the system dynamics and on the step length $\alpha$. In this case no information on the system dynamics is exploited and further analysis are required to provide a proof of convergence.

At each iteration of the algorithm, one could plot the achieved input-output relationship for each control variable to evaluate how they are modified. The purpose of this work is to obtain a controller ready to be used, hence one can perform a least squares minimization to fit a map on the new achieved input-output relationships. This is illustrated in the next section.

\section{Simulations}

In Figure 2 the evolution of the figure of merit $J$ over different iterations $k$ is shown and the optimal results, for the 28-th iteration, are summarized in Table I, where $t_{0}=5$ $\mathrm{s}$ and $t_{f}=120 \mathrm{~s}$. In Figure 3 it is posible to see the improvement in the torque tracking performance, at the reasonable cost of a slightly increase in the fuel consumption as visible in Figure 4.

As already stated in section III, the outcome of ILC is a control signal $u_{k}(t)$ which has to be realized in a controller to be used on different torque reference profiles too. This has been done with a least squares spline fitting over the $T^{o} \rightarrow u_{j}$ relations, for $j=1 \ldots m$, resulting in a piecewise polynomial which has been used to compute the updated maps $\mathcal{M}_{i}^{L S}$. In Figure 5, 6, 7 it is possible to compare the original calibration maps $\mathcal{M}_{i}$, the ones obtained from the ILC procedure, denoted by $\hat{\mathcal{M}}_{i}$, and the maps $\mathcal{M}_{i}^{L S}$.

The torque tracking performance has been also assessed when using the maps $\mathcal{M}_{i}^{L S}$. Results are shown in Figure 8 and in Table II. The minimum value achieved by the ILC iterations is slightly increased when the fitted maps are applied. This is due to the fact that the optimal input signal resulting from ILC is different from its least square average, as it is shown in Figures 5, 6, 7. Despite that, the tracking achieved by means of the fitted maps is significantly better with respect to the tracking achieved by the original maps. Notably the achieved maps, as well as the ILC control, avoid

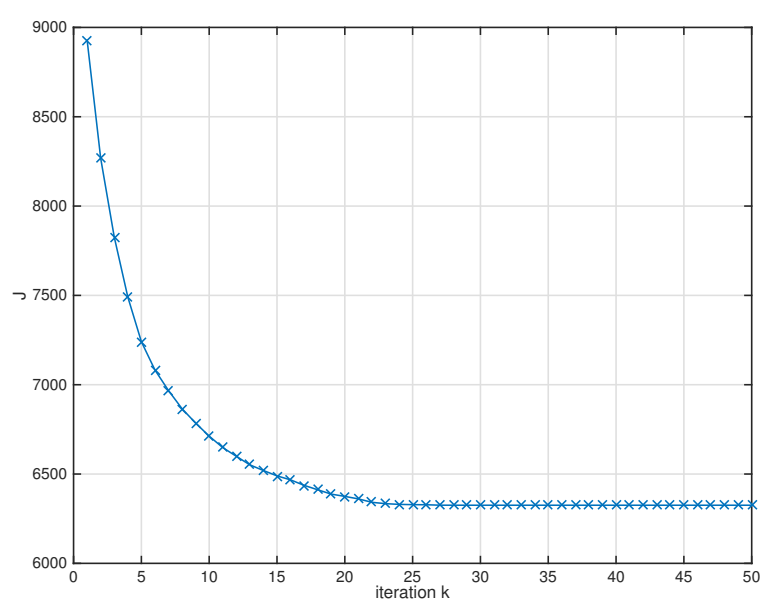

Fig. 2. Figure of merit evolution with gradient descent algorithm. The minimum value has been obtained for $k=28$ and corresponds to $J=$ 6325.4 .

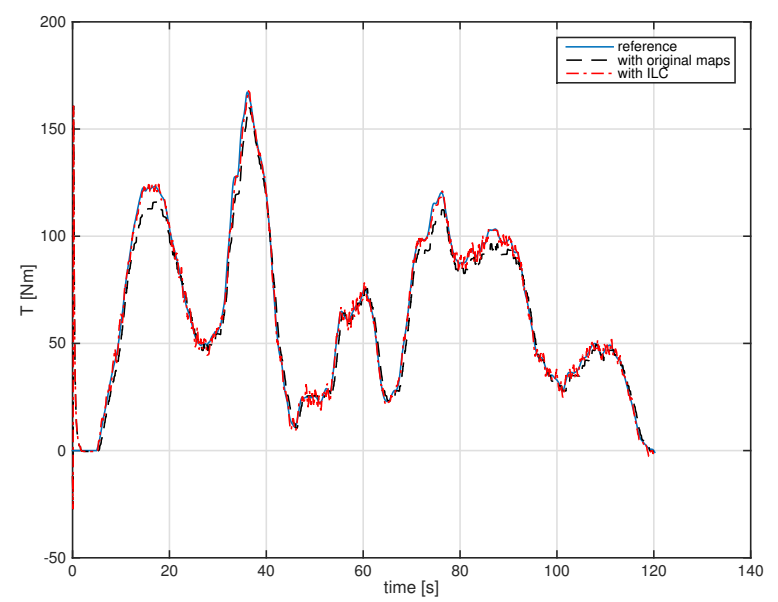

Fig. 3. Tracking of the reference torque (in solid blue) achieved with ILC (in dot-dashed red) and with the original maps (in dashed black).

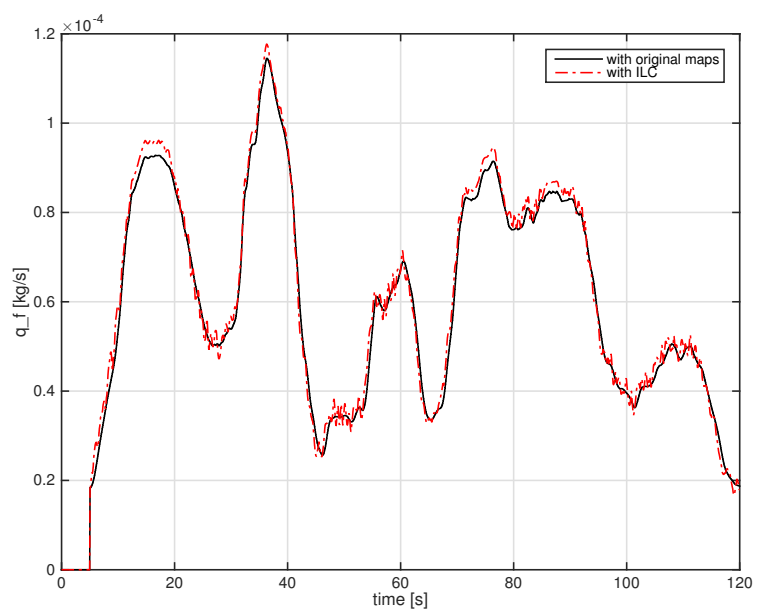

Fig. 4. Fuel consumption $q_{f}$ achieved with the original maps (in solid black) and with ILC (in dot-dashed red). 


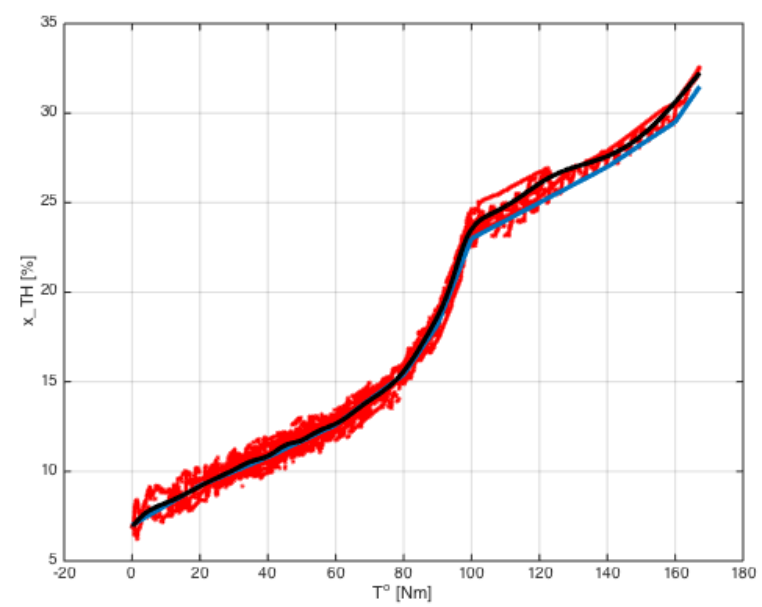

Fig. 5. Maps from $T^{o}$ to $x_{T H}$ : in blue solid thin line the original map $\mathcal{M}_{1}$; in red dots the map $\hat{\mathcal{M}}_{1}$ resulting from the ILC process; in black solid bold line the map $\mathcal{M}_{1}^{L S}$ obtained from a least squares piecewise polynomial fitting of the optimal map.

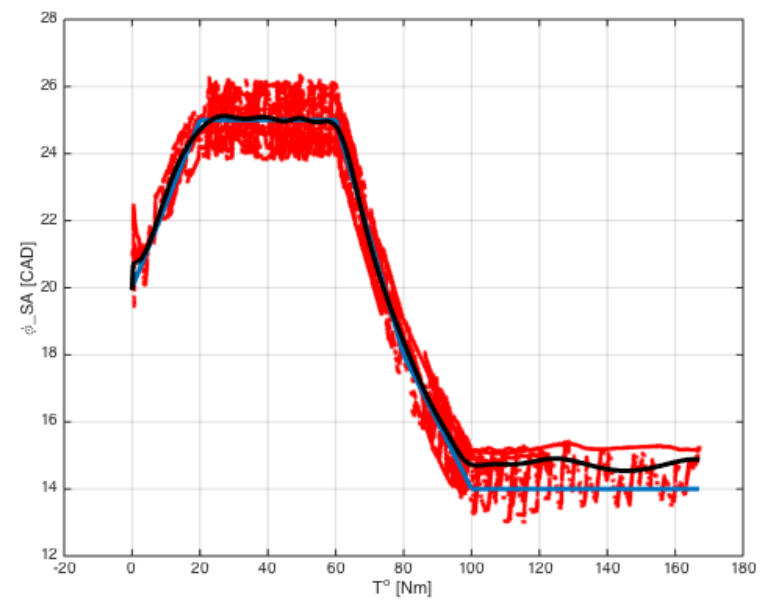

Fig. 6. Maps from $T^{o}$ to $\phi_{S A}$ : in blue solid thin line the original map $\mathcal{M}_{2}$; in red dots the map $\hat{\mathcal{M}}_{2}$ resulting from the optimization process; in black solid bold line the map $\mathcal{M}_{2}^{L S}$ obtained from a least squares piecewise polynomial fitting of the optimal map.

knocking and misfiring, while mantaining the control signals inside the bounding region, see Figure 9, 10, 11 and 12.

In order to provide a cross-validation for the proposed approach, the achieved control maps are exploited to track a new torque reference profile, different from the torque reference considered in the design of the control maps by means of the ILC. The results are showed in Figure 13 and summarized in Table II: as one can see by means of the designed control maps a good tracking is achieved for the new reference torque and, although not shown for space reasons, knocking and misfiring are avoided and control bounds are respected.

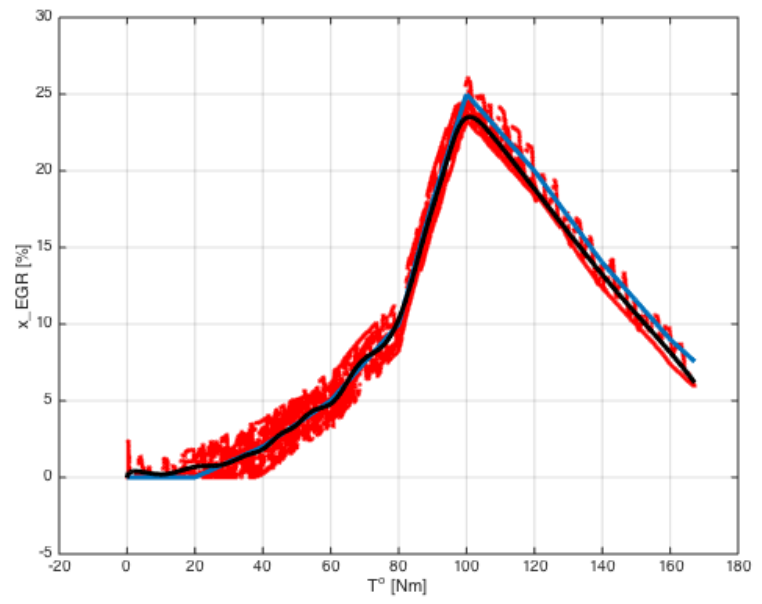

Fig. 7. Maps from $T^{o}$ to $x_{E G R}$ : in blue solid thin line the original map $\mathcal{M}_{3}$; in red dots the map $\hat{\mathcal{M}}_{3}$ resulting from the optimization process; in black solid bold line the map $\mathcal{M}_{3}^{L S}$ obtained from a least squares piecewise polynomial fitting of the optimal map.

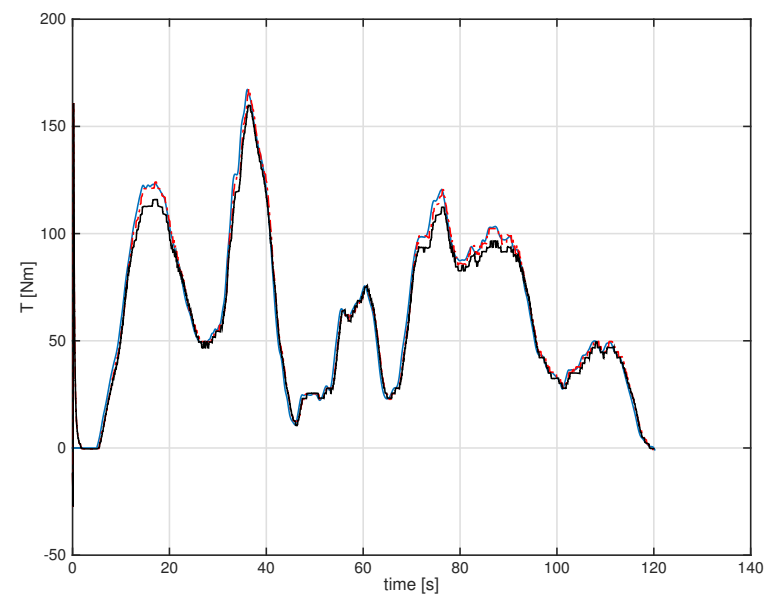

Fig. 8. Tracking of the reference torque (in solid blue) achieved with the new least squares fitted maps $\mathcal{M}_{i}^{L S}$ (in dot-dashed red) and with the original maps (in solid black).

TABLE I

PERFORMANCE OBTAINED WITH ILC OPTIMAL CONTROL

\begin{tabular}{|l||l|l|l|}
\hline Objectives & Equation & $\begin{array}{l}\text { Value } \\
\text { (original) }\end{array}$ & $\begin{array}{l}\text { Value } \\
\text { ILC }\end{array}$ \\
\hline $\begin{array}{l}\text { Fuel } \\
\text { consumption }\end{array}$ & $J_{f u e l}=\int_{t_{0}}^{t_{f}} c_{t} q_{f}(\tau) d \tau$ & 5621.6 & 5721.3 \\
\hline $\begin{array}{l}\text { Torque } \\
\text { deviation }\end{array}$ & $J_{T}=\int_{t_{0}}^{t_{f}} c_{t}\left(T^{o}(\tau)-T(\tau)\right)^{2} d \tau$ & 3307.3 & 604.1 \\
\hline $\begin{array}{l}\text { Overall } \\
\text { objective }\end{array}$ & $J=J_{f u e l}+J_{T}$ & 8928.9 & 6325.4 \\
\hline
\end{tabular}



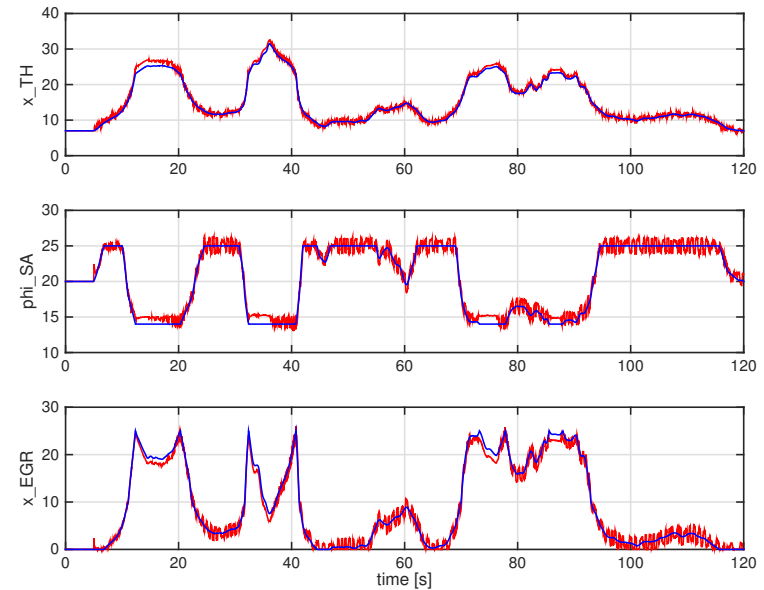

Fig. 9. Control signals: in blue line the signals obtained with the original maps, in red line the signals obtained from the ILC controller.
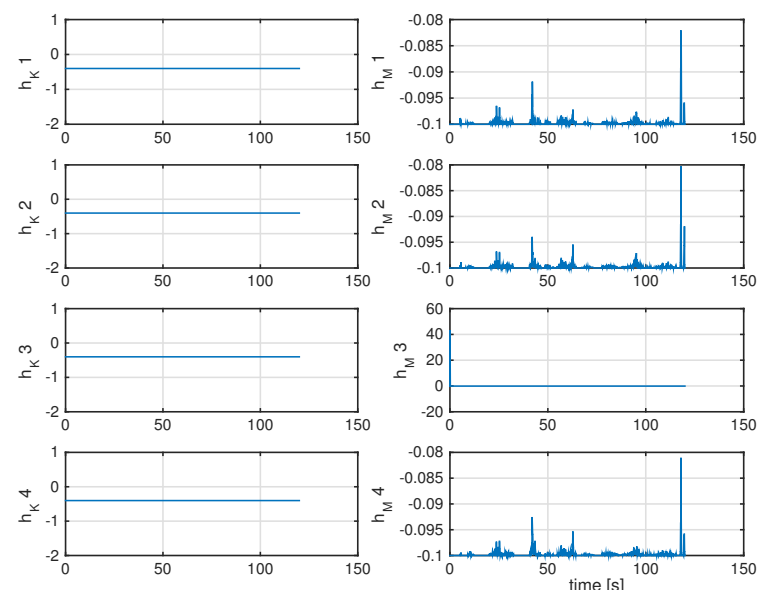

Fig. 10. Constraint function h: it is shown for all the cylinders with the ILC optimal control.
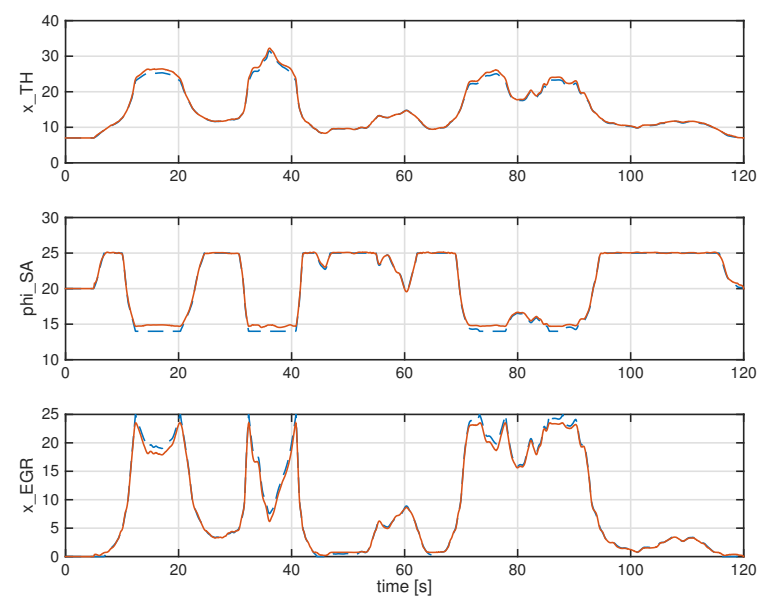

Fig. 11. Control signals: in blue line the signals obtained with the original maps, in red line the signals obtained from the new calibration maps.

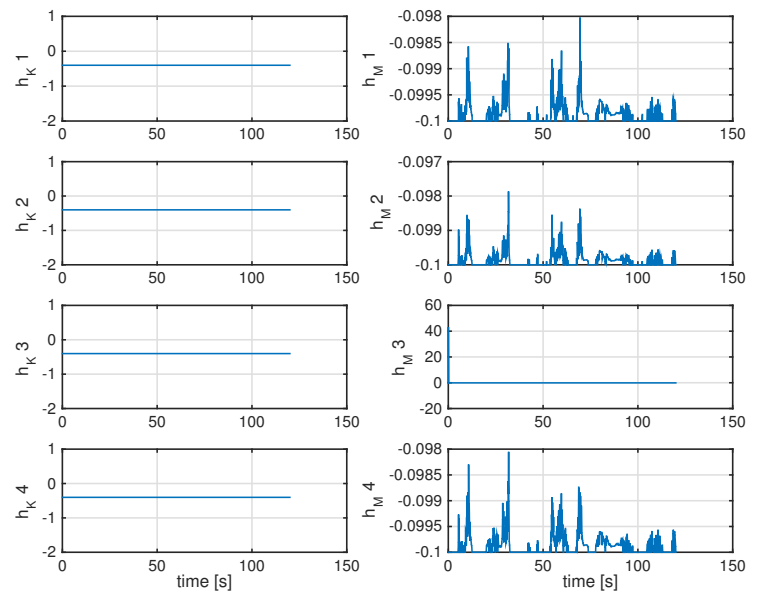

Fig. 12. Constraint function h: it is shown for all the cylinders with the new calibration maps.

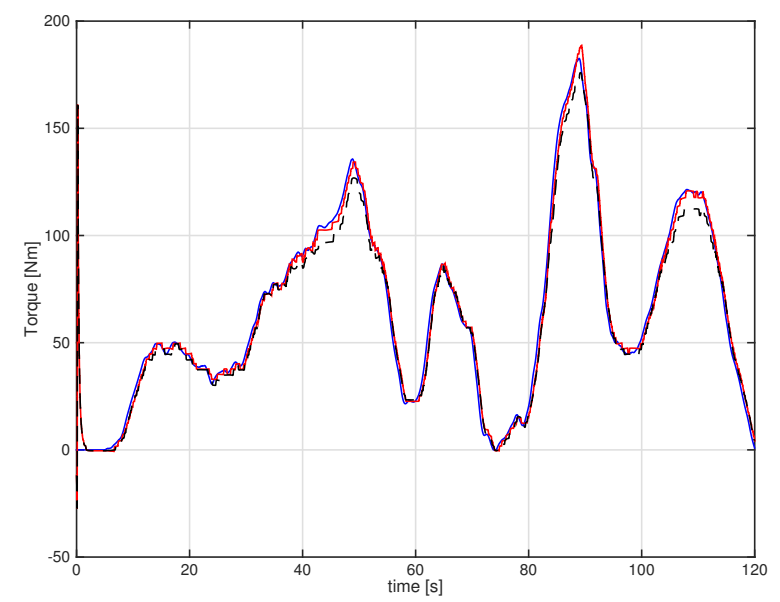

Fig. 13. Tracking of a different reference torque (in solid blue) achieved with the new least squares fitted maps $\mathcal{M}_{i}^{L S}$ (in solid red) and the original maps (in dashed black).

TABLE II

PERFORMANCE OBTAINED

\begin{tabular}{|l||l|l|l|}
\hline Objectives & $\begin{array}{l}\text { Value new } \\
\text { maps (same } \\
\text { reference of } \\
\text { Table I) }\end{array}$ & $\begin{array}{l}\text { Value cross- } \\
\text { validation, } \\
\text { original } \\
\text { maps }\end{array}$ & $\begin{array}{l}\text { Value cross- } \\
\text { validation, } \\
\text { new maps }\end{array}$ \\
\hline Fuel consumption & 5719.8 & 5591.0 & 5695.5 \\
\hline Torque deviation & 1556.6 & 3539.8 & 1912.2 \\
\hline Overall objective & 7276.4 & 9130.9 & 7607.7 \\
\hline
\end{tabular}




\section{CONCLUSION}

In the context of a student competition, a method for optimal tuning of ECU calibration maps is developed. The proposed algorithm provides improved calibration maps that have been tested on a given torque profile reference and cross-validated on other torque profiles obtained from commonly used driving cycles. The promising results obtained in simulation motivate further investigation, both theoretical and experimental, to understand how the Iterative Learning Control paradigm can be used to improve current state of the art Engine Control Units. Also it is of interest to investigate how such methods could be used in on-line operation of the engines to automatically update the calibration maps over time, refining the engines performances despite degradation of its components.

\section{REFERENCES}

[1] Competition specifics: http://www.ecc15.at/media/ecc15_ student_competition.pdf.

[2] Freeman, C.T.; Tan, Y.; Iterative Learning Control With Mixed Constraints for Point-to-Point Tracking, IEEE Trans. Control Systems Technology, Vol. 21, No. 3, pp. 604-616, May 2013.

[3] Arimoto, S.; Kawamura, S.; Miyazaki, F., Bettering operation of robots by learning, Journal of Robotic systems, vol.1, no.2,pp.123-140, 1984.

[4] Bristow, D.A.; Tharayil, M.; Alleyne, A.G., A survey of iterative learning control, Control Systems, IEEE, vol.26, no.3, pp.96-114, June 2006.

[5] Guzzella, L.; Onder, C., Introduction to Modeling and Control of Internal Combustion Engine Systems, 2nd ed., 2010, Springer.

[6] Kampelmühler, F. T.; Paulitsch, R.; Gschweitl, K. Automatic ECUCalibration-An Alternative to Conventional Methods. SAE Technical Paper, 1993.

[7] Del Re, L.; Allgwer, F.; Glielmo, L.; Guardiola, C.; Kolmanovsky, I., Automotive model predictive control: models, methods and applications, Vol. 402, 2010, Springer.

[8] Miotti, A., Models and control strategies for automotive propulsion systems, PhD Thesis, 2007. 